Gefässchirurgie 2014 • 19:698-700

DOI 10.1007/s00772-014-1379-x

Online publiziert: 7. Dezember

๑) Springer-Verlag Berlin Heidelberg 2014

M.K. Widmer $\cdot$ T. Windisch

Universitätsklinik für Herz-und Gefässchirurgie, Inselspital, Bern

\title{
Die Qual der Wahl beim Dialysezugang
}

Die Anzahl Patienten mit chronischer Nierenerkrankung steigt mit der Zunahme der durchschnittlichen Lebenserwartung. Ebenso nimmt Diabetes mellitus als Ursache für das Nierenversagen zu. Ein neuer Bericht aus den USA bestätigt zudem den bekannten Umstand, dass $\mathrm{Pa}$ tienten mit einem Hämodialysekatheter ein weit höheres Risiko für Tod, Infekte mit Todesfolge und kardiovaskuläre Ereignisse gegenüber Patienten mit arteriovenösen Fisteln (AVF) oder Shunts aufweisen [20]. Neueste Zahlen belegen, dass nach wie vor bei zu vielen Patienten das Nierenersatzverfahren über einen zentralvenösen Hämodialysekatheter begonnen wird [29]. Anstrengungen, die Anzahl der Neuanlagen von AVF zu erhöhen, haben dazu geführt, dass an Kongressen häufiger über das Problem einer fehlenden Fistelreifung diskutiert wird. Angesichts der genannten Probleme ist der primäre Einsatz einer Kunststoffprothese wieder ein Thema. Die Industrie hat seit der ersten Implantation einer PTFE-Prothese zur Hämodialyse im Jahre 1976 immer wieder modifizierte Grafts auf den Markt gebracht, um deren Offenheitsrate gegen- über der AVF zu verbessern [1]. Zu bemängeln bleibt, dass die Evidenzlage hinsichtlich solcher Prothesen oftmals wegen kleiner Fallzahlen als eher schlecht zu bewerten ist [11]. Zudem erschweren Studien mit Prothesenimplantationen an verschiedenen Lokalisationen die Vergleichbarkeit. Mittlerweile lassen sich Gruppen von verschiedenen Prothesentypen bilden, die sich durch gewisse Merkmale unterscheiden (• Tab. 1).

Nach Sichtung der Literatur lassen sich folgende Punkte zusammenfassen:

- Die Bewertung bezüglich der Offenheitsrate von Prothesen mit geänderter Geometrie ist offen.

- Prothesen mit einem Cuff haben in zwei Studien bessere Offenheitsraten gezeigt als herkömmliche Prothesen [15, 23].

- Für eine Prothese, die durch ihre Konfiguration von Spiralen einen laminaren Blutfluss erzeugt, gibt erst vorläufige Kongressmitteilungen von kleinen Serien [10].

- Die abschließende Beurteilung über den Effekt von heparinbeschichteten Prothesen ist ebenfalls offen, da im Namen der Herausgeber, der Schriftleitung, des Editorial Boards und aller Freunde der Gefässchirurgie wünsche ich Ihnen ein friedliches Weihnachtsfest und alles Gute, Gesundheit, Erfolg und Zufriedenheit für das Neue Jahr.

Mit den besten Grüßen

Ihr

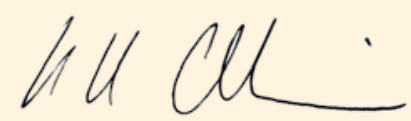

H.H. Eckstein
Tab. 1 Klassifikation von Prothesentypen

\begin{tabular}{|ll}
\hline Art von Prothesen & Ziel der Veränderung \\
\hline Kunststoffprothesen (PTFE, Polyurethan) & - \\
\hline Prothesen mit veränderter Geometrie & Bessere Offenheit, Verhinderung einer MIH \\
\hline Beschichtete Prothesen (Heparin, Silber) & Bessere Offenheit, geringere Infektrate \\
\hline Früh anstechbare Prothesen & Vermeidung von HD-Kathetern \\
\hline Hybridprothesen & $\begin{array}{l}\text { Zugang bei schwieriger Anatomie, Verhinderung einer } \\
\text { MIH }\end{array}$ \\
\hline Biologische Prothesen & Geringere Infektrate, bessere Offenheit? \\
\hline Gewebsgezüchtete Prothesen & Bessere Offenheit, geringere Infektrate \\
\hline MIH myointimale Hyperplasie & \\
\hline
\end{tabular}


in zwei nicht randomisierten Studien gegensätzliche Ergebnisse bezüglich Offenheit gezeigt wurden $[2,5]$.

- Früh anstechbare Prothesen aus Polyurethan oder Polytetrafluoroethylen (PTFE), zum Teil in Kombination mit einem Elastomer, werden heute immer häufiger eingesetzt, weil man diese bereits 12 bis $24 \mathrm{~h}$ nach der Implantation anstechen kann $[3,4,6,9$, 12, 21, 26]. Damit lassen sich zentralvenöse Hämodialysekatheter umgehen. Es gibt aber nur wenige Studien mit niedrigen Fallzahlen zu diesen Prothesen. Die Offenheitsrate ist vergleichbar mit herkömmlichen Prothesen. Bisher wurden auch keine höheren Raten von Wundkomplikationen und Infekten berichtet, obwohl ein solches Risiko wegen der nicht abgeschlossenen Wundheilung zum Zeitpunkt der Punktionen denkbar wäre.

- Bei den Hybridprothesen, die sowohl endovaskulär als auch offen implantiert werden, fehlen ebenfalls verlässliche Langzeitresultate $[8,13,14,16$, 24].

- Für den HeRo-Katheter, bei dem man einen PTFE-Graft mit einer zentralvenös eingeführten Kanüle verbindet, sind die Offenheitsraten vergleichbar mit den üblichen Prothesen [7, 17].

- Die Zahl der Anbieter für Bioprothesen ist in den letzten Jahren zurückgegangen, obwohl diesem Prothesenmaterial wegen der möglichen Infektresistenz ein gewisses Potenzial inne liegt $[18,22,25]$. Erste Fallberichte über Prothesen, deren Wandgewebe/material aus menschlichen Zellen gezüchtet wurde, sind viel versprechend $[19,27,28]$. Bis zur Serienreife werden aber noch Jahre vergehen. Was solche Prothese kosten werden, steht noch in den Sternen geschrieben.

\section{Fazit}

Mehr denn je müssen heute für jeden $\mathrm{Pa}$ tienten individuelle Entscheidungen darüber gefällt werden, welche Art von Dialysezugang gewählt werden kann/soll. Neben dem biologischen Alter, der Lebenserwartung, den Risikofaktoren und den anatomischen Gegebenheiten entscheidet der Zeitpunkt der Hämodialyse darü- ber, welcher Gefäßzugang sinnvoll ist und ob eine Kunststoffprothese eine vertretbare Option sein könnte.

In der vorliegenden Ausgabe der $\mathrm{Ge}$ fässchirurgie werden die Wichtigkeit der regelmäßigen klinischen Shuntkontrollen und die Vorteile der apparativen Flussmessung zur Verhinderung von Shuntthrombosen dargelegt. Zwei Beiträge befassen sich mit den heute empfohlenen Behandlungsstrategien von peripheren und zentralen Stenosen von arteriovenösen Fisteln. Für den Gefäss- und Shuntchirurgen ist es wichtig, über das Thema der Kalziphylaxie Bescheid zu wissen, da dieser Stoffwechselstörung mit ihren Folgen im klinischen Alltag eine große Bedeutung zukommt.

Mit der Kasuistik „Shuntchirurgisches Kaleidoskop“ starten wir den Versuch, interaktiv Indikationen und Therapiestrategien mit der Leserschaft zu diskutieren und zugleich zu erfahren, wie sich Shuntchirurgen außerhalb von Mitteleuropa in einer schwierigen Situation entscheiden. Wir rufen alle Leser auf, eigene Fälle oder größere Fallserien in unserer Zeitschrift zur Diskussion zu stellen, damit die Shuntchirurgie innerhalb der Gefäßchirurgie präsenter wird.

Viel Freude bei der Lektüre

Ihr



Matthias Widmer

\section{Korrespondenzadresse}

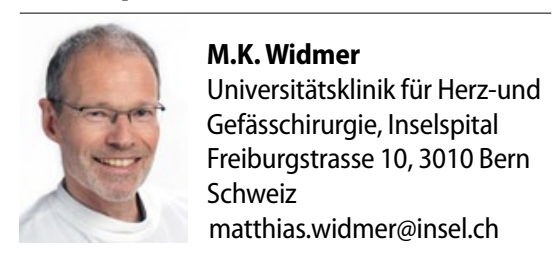

Interessenkonflikt. M.K. Widmer weist auf folgende Beziehungen hin: Referententätigkeit für die Firmen Atrium/Maquet und Bionova. T. Windisch gibt an, dass kein Interessenkonflikt besteht.

\section{Literatur}

1. Baker LD Jr, Johnson JM, Goldfarb D (1976) Expanded polytetrafluoroethylene (PTFE) subcutaneous arteriovenous conduit: an improved vascular access for chronic hemodialysis. Trans Am Soc Artif Intern Organs 22:382-387

2. Charlton-Ouw KM, Nosrati N, Miller CC, $3^{\text {rd }}$ et al (2012) Outcomes of arteriovenous fistulae compared with heparin-bonded and conventional grafts for hemodialysis access. J Vasc Access 13(2):163167

3. Chemla ES, Nelson S, Morsy M (2011) Early cannulation grafts in straight axillo-axillary angioaccesses avoid central catheter insertions. Semin Dial 24(4):456-459

4. Chiang N, Hulme KR, Haggart PC, Vasudevan T (2014) Comparison of FLIXENE and standard PTFE arteriovenous graft for early haemodialysis. J Vasc Access 15(2):116-122

5. Davidson I, Hackerman C, Kapadia A, Minhajud$\operatorname{dib}$ A (2009) Heparin bonded hemodialysis e-PTFE grafts result in $20 \%$ clot free survival benefit. The journal of vascular access 10:153-156

6. Ferraresso M, Bertoli S, Nobili P, Bortolani EM (2013) Early experience with a newly developed electrospun polycarbonate-urethane vascular graft for hemodialysis access. The journal of vascular access 14:252-256

7. Gage SM, Katzman HE, Ross JR et al (2012) Multicenter experience of 164 consecutive Hemodialysis Reliable Outflow [HeRO] graft implants for hemodialysis treatment. Eur J Vasc Endovasc Surg 44(1):93-99

8. Glickman MH (2011) HeRO Vascular Access Device. Semin Vasc Surg 24:108-112

9. Glickman MH, Stokes GK, Ross JR et al (2001) Multicenter evaluation of a polytetrafluoroethylene vascular access graft as compared with the expanded polytetrafluoroethylene vascular access graft in hemodialysis applications. J Vasc Surg 34(3):465-472; discussion 472-463

10. Inston N. Hoffmann WJ (2013) Early experience with the SLF (Spiral Laminar Flow) AV Access Graft. J Vasc Access 14 (1):43

11. Inston NG, Jones R (2014) Devices in vascular access: is technology overtaking evidence? J Vasc Access 15(2):73-75

12. Karatepe C, Aitinay L, Yetim TD et al (2013) A novel electrospun nano-fabric graft allows early cannulation access and reduces exposure to central venous catheters. J Vasc Access 14(3):273-280

13. Katzman HE, McLafferty RB, Ross JR et al (2009) Initial experience and outcome of a new hemodialysis access device for catheter-dependent patients. J Vasc Surg 50(3):600-607

14. Klee U, Mistry H, Brar R, Valenti D (2012) Innovationen von Dialyseshuntprothesen. Gefässchirurgie 17:106-114

15. Ko PJ, Liu YH, Hung YN, Hsieh HC (2009) Patency rates of cuffed and noncuffed extended polytetrafluoroethylene grafts in dialysis access: a prospective, randomized study. World J Surg 33(4):846-851

16. Mistry H, Stephenson MA, Valenti D (2013) Early outcomes of the intraluminal flow guard device for secondary renal access. JVasc Access 14(2):131134

17. Nassar GM, Glickman MH, McLafferty RB et al (2014) A comparison between the HeRO graft and conventional arteriovenous grafts in hemodialysis patients. Semin Dial 27(3):310-318 
18. Palumbo R, Niscola P, Calabria S et al (2009) Longterm favorable results by arteriovenous graft with Omniflow II prosthesis for hemodialysis. Nephron Clin Pract 113(2):c76-80

19. Peck MK, Dusserre N, Zagalski K et al (2011) New biological solutions for hemodialysis access. J Vasc Access 12(3):185-192

20. Ravani P, Palmer SC, Oliver MJ et al (2013) Associations between hemodialysis access type and clinical outcomes: a systematic review. J Am Soc Nephrol 24(3):465-473

21. Schild AF, Schuman ES, Noicely K et al (2011) Early cannulation prosthetic graft (Flixene) for arteriovenous access. J Vasc Access 12(3):248-252

22. Sert S, Demirogullari B, Ziya Anadol A et al (2000) Bovine and PTFE vascular graft results in hemodialysis patients. JVasc Access 1(4):148-151

23. Sorom AJ, Hughes CB, McCarthy JT et al (2002) Prospective, randomized evaluation of a cuffed expanded polytetrafluoroethylene graft for hemodialysis vascular access. Surgery 132(2):135-140

24. Steerman SN, Wagner J, Higgins JA et al (2013) Outcomes comparison of $\mathrm{HeRO}$ and lower extremity arteriovenous grafts in patients with longstanding renal failure. J Vasc Surgery 57(3):776783; discussion 782-773

25. Tahami VB, Hakki H, Reber PU et al (2007) Polytetrafluoroethylene and bovine mesenterial vein grafts for hemodialysis access: a comparative study. JVasc Access 8(1):17-20

26. Tozzi $M$, Franchin $M$, letto $G$ et al (2014) Initial experience with the Gore ${ }^{\circledast}$ Acuseal graft for prosthetic vascular access. J Vasc Access doi: 10.5301/ jva.5000276

27. Wystrychowski W, Cierpka L, Zagalski K et al (2011) Case study: first implantation of a frozen, devitalized tissue-engineered vascular graft for urgent hemodialysis access. J Vasc Access 12(1):67-70

28. Wystrychowski W, McAllister TN, Zagalski K et al (2013) First human use of an allogeneic tissue-engineered vascular graft for hemodialysis access. J Vasc Surg doi: 10.1016/j.jvs.2013.08.018

29. Xue $H, I x ~ J H$, Wang W et al (2013) Hemodialysis access usage patterns in the incident dialysis year and associated catheter-related complications. Am J Kidney Dis 61(1):123-130

\section{Inflammation}

Neue Therapieansätze in der kardiovaskulären Medizin

Gegenwärtig beschränken sich die Therapiestrategien bei atherosklerotischen Erkrankungen auf die Behandlung kardiovaskulärer Risikofaktoren wie Hypertonie, Hyperlipidämie und Diabetes mellitus und die Revaskularisation durch kathetergestützte oder operative Verfahren. Experimentelle Studien der letzten Jahre haben uns allerdings tiefe Einblicke in die pathomechanistischen Grundlagen der Atherogenese gewährt. Sie lehren uns, dass es sich um eine chronisch entzündliche Erkrankung handelt, bei der die Einlagerung von Lipiden in der Gefäßwand die Einwanderung und Vermehrung von Entzündungszellen bewirkt, die ihrerseits das Plaquewachstum fördern und die Plaque destabilisieren können. Dies findet klinischen Ausdruck in einem erhöhten hochsensitiven C-reaktiven Protein (hsCRP) bei Patienten mit kardiovaskulärer Erkrankung. Bei gesunden Individuen mit normwertigem Cholesterin führt bereits ein erhöhtes hsCRP zu einem erhöhten kardiovaskulären Risiko. Die Behandlung mit Statinen reduziert bei diesem Kollektiv die Ereignisrate sogar unabhängig von der Lipidsenkung, was die pleiotrope anti-inflammatorische Wirkung dieser Substanzen unterstreicht. Derzeit werden im Rahmen klinischer Studien antiinflammatorische Therapieansätze wie etwa der Folsäureantagonist Methotrexat (CIRT Studie) sowie der Interleukin-1\&\#61538; blockierenden Antikörper Canakinumab (CANTOS Studie) in großen Studien getestet.

Im Rahmen des kommenden Jahreskongresses der GTH, welcher vom 24.-27.02.2015 in Düsseldorf stattfindet, wird kardiovaskuläre Inflammation einen wesentlichen thematischen Schwerpunkt darstellen und durch international hochkarätige Wissenschaftler und Kliniker dargestellt werden.

Quelle: Prof. Dr. A. Zirlik vom UniversitätsHerzzentrum Freiburg

\section{Neue Leitlinien zur Behandlung von Aorten-Erkrankungen}

Die European Society of Cardiology (ESC) veröffentlichte neue Leitlinien zur Behandlung von Aorten-Erkrankungen, die standardisierte Hilfestellung zu mehr Aorta-Krankheiten als bisherige Leitlinien umfassen. So berücksichtigen sie auch Bauchaortenaneurysmen, Aorten-Entzündungen, Aorten-Tumoren und angeborene Erkrankungen sowie Erbkrankheiten der Aorta. Erstmals enthalten ist zudem ein Flussdiagramm zur Entscheidungsfindung beim ",akuten Aorten-Syndrom", welches die behandelnden Ärzte bei der Diagnosestellung und Auswahl der Behandlungsschritte unterstützt. Weiterhin empfehlen die Spezialisten der ESC die Einrichtung von eigenen Aorten-Zentren in Krankenhäusern mit Fachärzten aus der Kardiologie, der Radiologie, der Herz- und Gefäßchirurgie, der Kinderkardiologie und der Genetik.

Die neuen Leitlinien sind vor allem auch durch die Fortschritte in der Bildgebung wie der Computertomographie und Magnetresonanztomographie möglich, die eine viel detailliertere Untersuchung der Hauptschlagader erlauben. So lässt sich mittlerweile die Aorta bei jedem Patienten mit Hilfe einer Software in 3D nachbilden, so dass auch die Gesamtstruktur der Aorta untersucht werden kann

Literatur: Erbel R, Aboyans V, Boileau C et al (2014) ESC guidelines on the diagnosis and treatment of aortic diseases. European Heart Journal, doi:10.1093/eurheartj/ehu281

Quelle: Universitätsklinikum Essen, www.uk-essen.de 